\title{
Preface
}

\section{PET and Al Trajectories Finally Coming into Alignment}

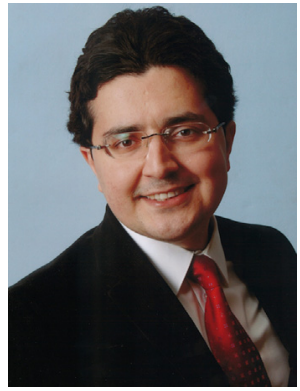

Babak Saboury, MD, MPH, DABR, DABNM

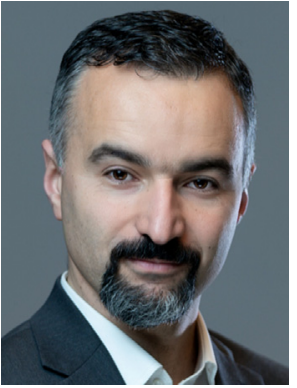

Arman Rahmim, PhD, DABSNM

Editors

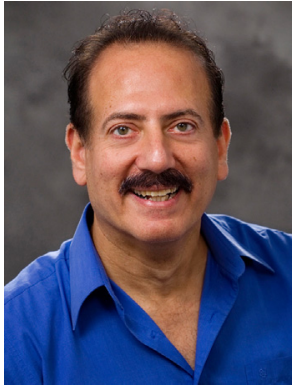

Eliot Siegel, MD
As two of the "hottest" and rapidly evolving areas of research and development in medicine, both with tremendous future potential and accelerating clinical applications in patient care, PET and Al (Artificial Intelligence) have been on a collision path for the past several years, but only recently have these fields begun to form a synergistic partnership. This first issue and a subsequent second issue in PET Clinics are planned to make up for lost time in the medical literature and address the emerging opportunities, challenges, and practical clinical implications of Al for PET. Like PET, the basic technologies underlying $\mathrm{Al}$ have existed for decades but have only recently been made practical by advances in hardware, specifically, the utilization of graphics-processing units during the past decade. These were applied with tremendous success to a challenge in computer vision resulting in a major leap forward that has enabled a new era of practical applications in image and speech recognition, near autonomous self-driving cars, language translation, advanced predictive modeling in a wide variety of disciplines, and high-profile success in board games, such as Chess and Go, Poker, and a variety of video games, all of which were previously thought to be within the sole domain of humans. The term Al has become synonymous with deep learning or convolutional neural networks within the past several years, while it continues to be also used in its original definition as the use of computer systems using algorithms to perform tasks that typically require human intelligence. The use of deep learning represents a fundamental paradigm shift from previous approaches in the detection and diagnosis of disease, as these algorithms are generated directly from large, annotated data sets without requiring the many handcrafted, painstaking steps typically required in the development of computer-aided detection and diagnosis algorithms. This generation of "computer code" directly from the data represents a means of rapidly turning large PET annotated data sets into a wide variety of different potentially useful clinically useful tools.

A variety of medical $\mathrm{Al}$ applications have recently emerged, most notably in medical imaging, such as detection of early signs of stroke on computed tomographic (CT) scans, assessment of delays in pediatric bone development, detection and assessment of lung nodules, and hundreds of other applications. Ironically, despite the early and successful adoption of computerized techniques and analytics in nuclear medicine, deep learning (Al) advances have focused much more on mammography, CT, MR imaging, and conventional radiography. This may in large part be due to greater availability to computer scientists and other Al researchers of large public databases with those modalities and relatively sparse availability of readily accessible nuclear medicine data sets. As an inherently multimodality imaging specialty, PET/CT offers an intriguing, inherent breadth of data from the combination of pixel data representing electron density (and in some cases dynamic contrast enhancement) with 
molecular activity, which can be assessed at a single or multiple time points. While CT scans have served as a common source of data for deep learning, neither PET-only images nor CT plus PET have been as widely utilized for Al applications (though there is a significant upward trend which this special issue covers).

The first issue of this special $\mathrm{Al}$ issue (consisting of 14 articles) has a technical focus, spanning the wide range of steps and aspects wherein Al can have significant presence. It will first delve into the history as well as "anatomy and physiology" underpinnings of $\mathrm{Al}$ (articles by Toosi and colleagues; Bradshaw and McMillan). Al has profound potential for in silico radiopharmaceutical development (article by Ataeinia and Heidari) as well as radiochemistry and radiochemical engineering (article by Webb and Scott). One of the fascinating things about deep learning is the ability to use it not only to analyze images but also to generate images using a sample database, enabling its application in image reconstruction, which is perhaps a "killer application" at the present time, offering the potential for decreased imaging times and patient doses and improved image quality (article by Gong and colleagues). In addition, Al application in improved attenuation and scatter correction (article by McMillan and Bradshaw), postreconstruction image enhancement (noise reduction and/or resolution enhancement; article by Liu and colleagues), and highthroughput segmentation (eg, tumors or normal organs; article by Yousefirizi and colleagues) are covered. Furthermore, both handcrafted and deep radiomics methods and applications have been on the rise (article by Orlhac and colleagues). An innovative application of $\mathrm{Al}$ is in total body kinetic modeling (article by Wang and colleagues). Similarly, Al has potential in refining and optimizing personalized radiopharmaceutical therapies (Theranostics; article by Brosch-Lenz and colleagues). Techniques for objectively evaluating Al-based imaging methods and for implementing them equitably using evidence-based practice are also discussed in two of the articles in this issue (articles by Jha and colleagues; Nooraie and colleagues). In addition to the technology being explored and discussed from a research and clinical practice perspective, the industry perspective which is, of course, critically important to practical implementation, is presented in this initial issue as well (article by Sitek and colleagues).

We wish to especially acknowledge the members of the SNMMI Al task force who both directly, through their contributions to this issue, and indirectly, through their insightful discussions, played a major role in crafting this two-part series.

Babak Saboury, MD, MPH, DABR, DABNM Department of Radiology and Imaging Sciences

Clinical Center

National Institutes of Health $(\mathrm{NIH})$ 9000 Rockville Pike Bethesda, MD 20892, USA

Department of Radiology Hospital of the University of Pennsylvania

Department of Computer Science \& Electrical Engineering University of Maryland, Baltimore County

Arman Rahmim, PhD, DABSNM Departments of Radiology and Physics University of British Columbia BC Cancer Research Institute 675 West 10th Avenue

Office 6-112

Vancouver, BC V5Z 1L3, Canada

Eliot Siegel, MD

Department of Radiology

University of Maryland School of Medicine 655 West Baltimore Street Baltimore, MD 21201, USA

E-mail addresses: babak.saboury@nih.gov (B. Saboury) arman.rahmim@ubc.ca (A. Rahmim) esiegel@umaryland.edu (E. Siegel) 